\title{
On frame rate and player performance in first person shooter games
}

\author{
Kajal T. Claypool · Mark Claypool
}

(C) Springer-Verlag 2007

\begin{abstract}
The rate at which frames are rendered in a computer game directly impacts player performance, influencing both the game playability and enjoyability. However, despite the importance of frame rate and the wide-spread popularity of computer games, to the best of our knowledge, there is little quantitative understanding of the effects of frame rate on player performance in computer games. This paper provides a unique classification of actions in First Person Shooter (FPS) games based on interaction requirements that allow qualitative assessment of the impact of frame rates on player performance. This qualitative assessment is supported by quantitative analysis from two large user studies that measure the effects of frame rate on the fundamental player actions in a FPS game. Nearly 100 users participated in the two user study experiments, providing performance and perception data over a range of frame rates commonly studied for video streaming and inclusive of frame rates found in many computer game platforms. In general, the analysis shows that actions that require precise, rapid response, such as shooting, are greatly impacted by degradations in frame rates, while actions with lower precision and response requirements, such as moving, are more tolerant of low frame rates. These insights into the effects of frame rates on player performance can guide players in their choice for game settings and new hardware purchases, and inform system designers in their development of new hardware.
\end{abstract}

\footnotetext{
K. T. Claypool $(\varangle)$

Oracle Corporation, Nashua, NH 03062 USA

e-mail: kajal.claypool@ oracle.com

M. Claypool

Department of Computer Science, Worcester Polytechnic Institute,

Worcester, MA 01609, USA

e-mail: claypool@cs.wpi.edu
}

\section{Introduction}

The growth in the computer game industry has pushed for innovation in hardware development from new desktop graphics hardware to powerful mobile and hand-held game devices in the quest to support compelling computer games. While many of the newest games require state-of-art hardware to deliver rich and immersive games, economics dictate that the same games will be played without the latest hardware. Computer games typically run on platforms with a range of processing and display capabilities, and a single game title may be released on PC, console and hand-held devices simultaneously. Even games released only for PCs must be effective over a considerable range of processing power and graphics card capabilities.

There are many factors that influence player performance, ${ }^{1}$ such as resolution, network latency, and frame rate. Careful study of each factor is an essential first step in determining the overall impact on game playability. There has been some work on the effects of network latency $[2,5-8,10,14,15]$ and resolution on games [4]. This paper focuses on frame rate, a key parameter that affects both how smooth a game looks (hence the overall game immersiveness), and the overall player performance. ${ }^{1}$ While maximizing the frame rate is desirable, an increased frame rate often comes at the expense of a lower resolution, making game images look less sharp. Moreover, the diversity of game hardware results in the same game being played over a range of frame rates. This forces game platform designers and game developers to combine the available capabilities of the hardware with an ad hoc

\footnotetext{
${ }^{1}$ In a First Person Shooter game, performance is measured by the number of times the player kills opponents and/or the number of times the player dies.
} 
understanding of the effects of frame rate on game play in an effort to provide for smooth or sharp computer games.

Much of the intuition for the effects of frame rates on games is based on studies of perceived quality for video $[1,13,19-21,23]$. These studies have examined the effects of frame rate on users passively watching streaming video, and some have even measured user ability to learn from the video $[9,11,16]$. These studies have found that users can tolerate a significant degradation in frame rate and still have satisfying video. However, watching video, even coupled with interactions such as in a video-conference or for the purpose of information recognition, does not have the same interaction requirements as do computer games. There are fewer studies $[3,12,17,18,22]$ that have examined the effects of frame rate on users actively engaged in an interactive media environment. These studies have generally found that user performance suffers under extremely low frame rates [under 4 frames per second (fps)], while frame rates as low as 4 or 5 fps can support acceptable performance. However, even these more interactive applications do not have the same response time requirements as do computer games.

Computer games are unique in that even within a single game the interaction requirements range from those of passive video (such as watching a cut-scene), all the way through intense interactions of rapid response to visual input (such as during a close-combat free-for-all). Thus, not all phases of a computer game are equally sensitive to degradations in frame rates. When the game is starting, maps and other game information is loaded from disk, and when the game is transitioning between maps there is little user interaction, hence little sensitivity to frame rate. When the game proceeds to the actual game play, arguably the most important and interesting aspect of computer games, the player interacts with the game world. The play phase requires different types and levels of interactions between the player and the game. A First Person Shooter (FPS) game, the subject of this paper, sometimes requires quick hand-eye coordination in moving the cross-hairs of a gun to target an opponent in intense one-on-one combat with high precision weapons, while other times requires strategic movement of teams of players and less frequent combat with lower precision weapons or even vehicles.

Because of this diversity of player actions, not all actions are equally impacted by frame rate. Some actions such as shooting a sniper rifle at a moving opponent are greatly impacted by frame rate, while other actions such as selecting a set of troops and moving them across a battlefield tend to be less sensitive to frame rate. This work contributes a novel categorization of the effects of frame rate on the performance of different player actions based on two salient action properties: the precision required to complete the action and the deadline by which the action must be completed. Qualitatively, performance for actions with high precision and tight deadlines tends to drop off sharply with degradations in frame rate, while actions with lower precision and loose deadlines are more tolerant of changes in frame rates.

This paper presents results of carefully designed user studies investigating the effects of frame rate on users playing a FPS game, a popular game genre. Custom maps were designed to allow repeated testing of the core aspects of FPS play-shooting at an opponent and movement through the virtual world. Test harnesses were developed to first collect demographic data for each user, and then cycle through the custom maps with different frame rates, collecting performance data and user perceptions. One-hundred users participated in two sets of experiments, providing a large enough base for statistical significance for most of the data analyzed. Analysis shows that shooting performance, a player action that requires high precision and tight deadlines, is more sensitive to low frame rates than is navigating turns during movement which has slightly lower precision and a looser deadline. There is little difference in the effects of frame rate on performance for different kinds or speeds of movement, such as walking, running and jumping. Moreover, the effects of frame rate are remarkably different for FPS games than for streaming video and other interactive media. In particular, for FPS games, frame rate significantly degrades performance at frame rates that are quite tolerable for streaming video and even other interactive applications.

The results presented in this paper provide a quantitative understanding of the effects of frame rate on game playability, making it useful for: (1) game players who need to be able to make informed decisions on graphic card purchases and for adjustments to game display settings, when appropriate; (2) hardware developers, including those designing graphics cards and console devices, to enable better targeting of hardware improvements to aspects of the display that matter; (3) designers of small, resource-constrained devices that must ensure that the right level of graphics capabilities are factored into the design decisions of a device.

The rest of this paper is organized as follows: Section 2 describes the phases and interactions in FPS games; Sect. 3 presents a categorization of user interactions along deadline and precision axes; Sect. 4 provides insights into the effects of frame rate and frame resolution and informs our hypotheses; Sect. 5 describes the custom software and experimental methodology used for our study; Sect. 6 analyzes the user data; Sect. 7 describes related work; Sect. 8 summarizes our conclusions; and Sect. 9 presents possible future work.

\section{First person shooters}

First Person Shooter games are a popular game genre wherein the player looks through the eyes of the avatar (the first person) and engages in combat, typically with ranged weapons 
Fig. 1 Game phases for Quake 3, a typical First Person Shooter (FPS). a Setup phase, b Play phase, c Transition phase

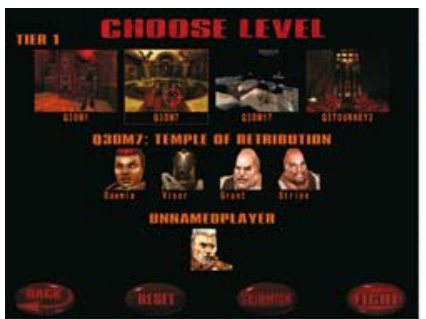

(a) Setup Phase

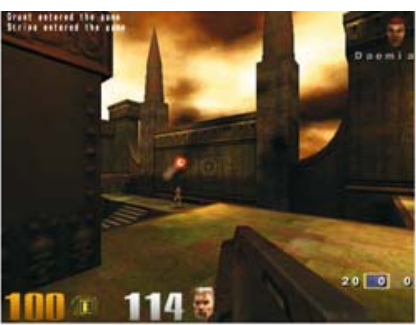

(b) Play Phase

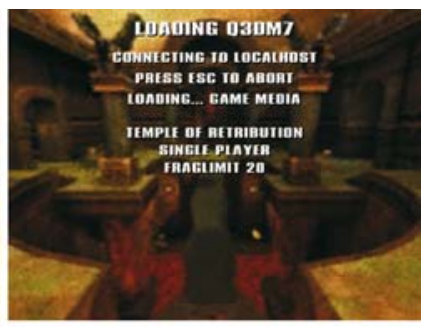

(c) Transition Phase (the shooter). FPS games, and most other games, go through several phases that differ in the player interactions with the game and, hence, in the impact of frame rate on the player performance. Although the duration and frequency of each phase varies depending upon the specific game, fundamental phases common to most FPS games include:

- Setup -During the Setup phase, players select the parameters specific for the gameplay they want at that time. For example, the player may select the map, avatar type and team. The Setup phase is generally relatively short, with fairly rapid player interactions so as to proceed on to gameplay as fast as possible. However, while players do interact with the game engine, the actions in this phase are mostly time-insensitive and so are unaffected by poor frame rates.

- Play-During the Play phase, the game is actually played, with players responding to the changes in the game state based on the visual images on the screen. For example, an FPS game responds to the player request for a movement of an avatar by visually altering the location of the avatar on the player's screen. The player may choose to respond by issuing a request to move the weapon sight to target an opponent or by issuing a new movement request for location change. The Play phase interactions are generally categorized by their time-critical nature. It is during the Play phase that the effects of frame rate on player actions are typically of most interest and it is the core subject of this paper.

- Transition - In between Play phases, most games have a Transition phase where game information is loaded and processed locally from a game disk into memory. For example, in an FPS the map may be loaded and the location of the powerups, weapons and opponents determined. The Transition phase generally has low frame rate requirements since there is no player interaction during this phase.

Figure 1 shows screenshots of the above phases for Quake 3, a typical FPS. Figure 1a shows the Setup phase for Quake 3 where the player can choose the map and opponents, Fig. 1b shows a Play phase with a free-for-all combat, and Fig. 1c
Table 1 Sensitivities for game phases for First Person Shooter games

\begin{tabular}{lll}
\hline Game phase & Time sensitivity & Frame rate sensitivity \\
\hline Setup & Low & Low \\
Play & Medium-high & Medium-high \\
Transition & Low & Low \\
\hline
\end{tabular}

shows a Transition phase where the map is being loaded before play starts.

In general, while the length and frequency of each phase can vary for different FPS games, the characteristics of the actions during each phase, and hence their sensitivity to frame rate, are consistent across different games. Table 1 summarizes the framerate and the time sensitivity for each phase.

\section{Frame rate and the play phase}

Low frame rates can reduce the feeling of game immersion and impact playability in all phases of the game. However, because of their interactive nature, player actions during the Play phase are most sensitive to degradations in frame rates.

Player actions in the Play phase of an FPS game can be classified primarily as shooting or movement. Shooting is defined as the task of identifying (sighting) a target via the placement of cross-hairs on the target and firing the weapon. Movement is defined as the task of navigating across a terrain to get from a start location to a destination location. A third hybrid action is a combination of movement and shooting requiring the player to sight a target and shoot while moving. While in general all FPS games have movement and shooting actions, the length and frequency of each action varies across FPS games, and sometimes even across maps for the same game. For example, id's Quake 3 typically features fast paced close combat fighting with rapid shooting and movement, while EA Games' Battlefield 1942 often features more strategic gameplay with longer periods of movement for positioning and shorter periods of intense shooting.

In general, actions in the Play phase for FPS games vary along two primary axes, deadline and precision. Deadline is the time required to complete the action, that is the length of time it takes to achieve the final outcome intended by the 
player. For example, in Quake 3, the deadline for shooting with railgun is the time it takes for the player to sight the opponent in the cross-hairs and to fire the weapon. Precision is the degree of accuracy required to complete the interaction successfully. For example, in Battlefield 1942, the precision of a sniper rifle targeting a distant enemy is the size of the opponent.

Shooting and movement actions have disparate deadline and precision requirements. This disparity can be observed both across the different actions (shooting versus movement) and even within an action (shooting with different types of weapons, or running versus walking). For example, shooting in FPS games generally has relatively high precision and tight deadline requirements, meaning the player must place the gun cross-hairs exactly on the enemy target to hit and the action must be carried out immediately or the enemy may move. However, the precision and deadline requirements for shooting can vary with the weapon used. For example, shooting with a sniper gun requires high precision with a tight deadline, shooting with a machine gun relaxes the precision since the gun has a scattered area effect, and shooting with a rocket launcher imposes relatively lower precision and deadline requirements than either the sniper gun or the machine gun. As another illustration, using a bazooka on an enemy tank has low precision and deadline because the target is large and the weapon has a large radius of effect and because the projectile takes some time to travel to the tank.

Movement in an FPS game requires high precision but has a relatively looser deadline than does shooting-the precise location will determine if a player's avatar can be hit, while moving from one location to another takes on the order of seconds. Precision and deadline requirements can vary across movement as well. For example moving on a twisty path has higher precision and deadline requirements than moving in a straight line.

Figure 2 shows a representation of the different player actions (shooting and movement) along the precision and

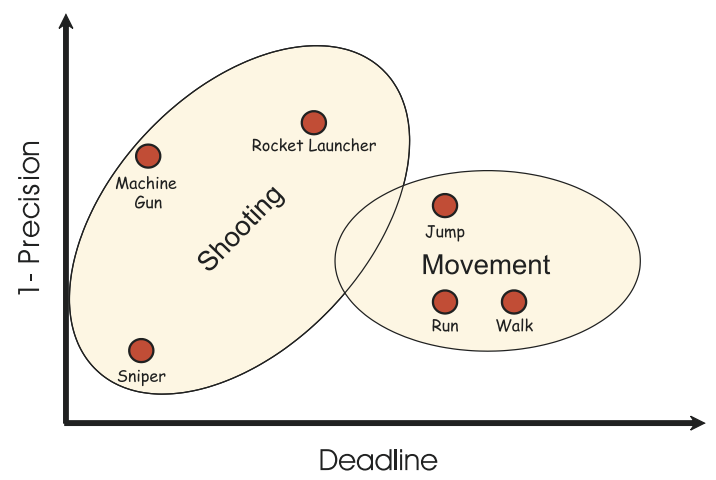

Fig. 2 Player actions in First person shooter classified along the precision and deadline axes deadline axes. The $x$-axis is the deadline requirement and the $y$-axis is amount of imprecision (indicated as 1-Precision). Shooting in general covers a region of high to medium precision with similar constraints on the deadline. Within the shooting region, an FPS Sniper has high precision and a tight deadline while an FPS Machine gun has lower precision and similar deadline requirements. Movement similarly covers a region that has high to medium precision, but its deadline requirements are typically looser in the range of medium to low deadline. Movement while running, for example, has medium precision and medium deadline requirements, while walking has similar precision but lower deadline requirements.

\section{Hypotheses}

The deadline and precision requirements for shooting and movement in a FPS game are directly correlated with the effects of frame rate on player performance for the particular action. In general, the further away an action is from the origin in the Precision-Deadline plane, the less the impact that frame rate has on player performance. Thus, as seen in Fig. 2, FPS Sniper is more sensitive to the frame rate than is FPS Machine gun, and movement while Running is more sensitive than movement while Walking.

Precision. Consider a shooting action where the player targets an opponent moving across the field of view from left to right, depicted in Fig. 3. With a high precision weapon, for example a sniper rifle, the player on the left sees the opponent as the solid outline, with the target circle representing the precision of the sniper gun the player is shooting (see Fig. 3a). At $60 \mathrm{fps}$, when the player aims and shoots the gun will hit any opponent within the circle. With lower frame rates the movement, and hence the location, of the opponent is not accurately relayed to the player. For example, at $7 \mathrm{fps}$ the player sees the opponent at the solid outline while the opponent has moved to the right to the dashed outline, resulting in a miss. However, when the player is shooting with a lowerprecision weapon, such as a machine gun, the target circle is larger (see Fig. 3b). In this case, while lower frame rates still delay the feedback of opponent position to the player, the opponent remains within the target area, enabling the player to score a hit.

This example illustrates our first insight: For a given game action, the higher the precision the greater the impact of frame rate on player performance.

Deadline. Consider once again the shooting action, and in particular the time it takes to sight the opponent in the cross-hairs, fire the weapon and have the projectile reach the opponent. If this time (the deadline) is short, such as 
Fig. 3 Shooting an opponent in a FPS Game. a High precision weapon, b low precision weapon

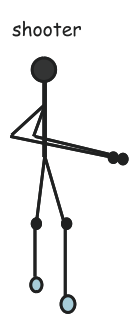

(a) High Precision Weapon
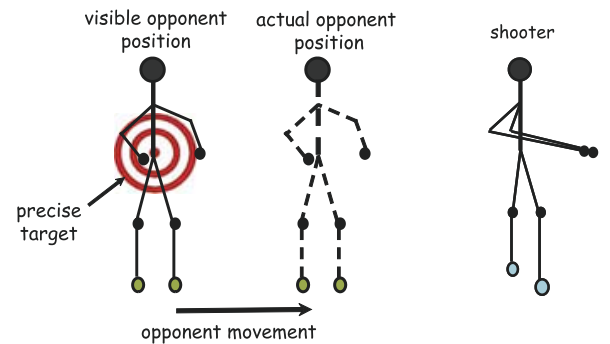

(b) Low Precision Weapon

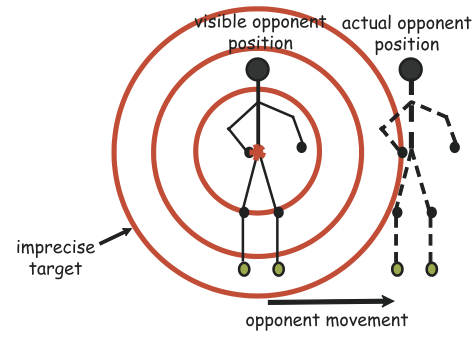

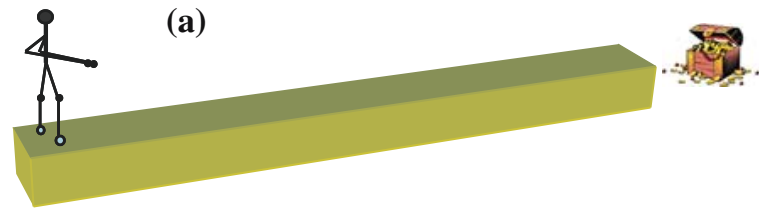

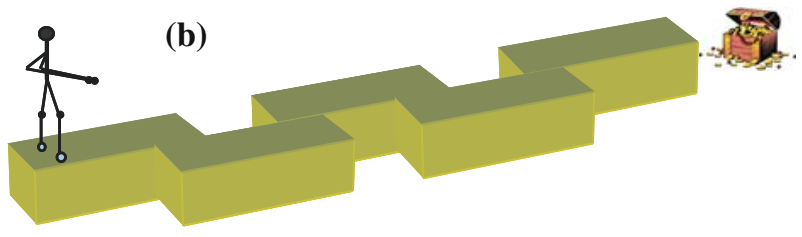

Fig. 4 Moving in a FPS Game. a Loose deadline, $\mathbf{b}$ tight deadline

for a sniper rifle, the delay in providing feedback to the player caused by low frame rate can be significant. However, when the deadline to complete the action is looser, the delay in providing user feedback is relatively less significant. For example, consider a movement action where the player must move along a suspended beam from point $\mathrm{A}$ to point B. If the beam is straight, as in Fig. $4 \mathrm{a}$, then the deadline is large relative to the player initiated command to move, so additional delays induced by a low frame rate do not impact performance much. However, if the beam is twisty, as in Fig. 4b, then there are many smaller move commands each with a tight deadline and even a small delay induced by low frame rates will significantly impede user movement or cause a fall.

This example illustrates our second insight: For a given game action, the tighter the deadline the greater the impact of frame rate on player performance.

\section{Methodology}

To corroborate the hypotheses laid out in Sect. 4 and to comprehensively evaluate the effect of frame rate on shooting and movement actions in general, the following methodology was employed:

- $\quad$ select a First Person Shooter game (see Sect. 5.1);

- build custom maps to enabled controlled experiments (see Sect. 5.2);
- construct test harnesses (see Sect. 5.3);

- setup a controlled environment to conduct the experiments (see Sect. 5.4);

- solicit users to participate in experiments (see Sect. 5.5).

\subsection{Quake 3}

All experiments were conducted using Quake 3 Arena (or Quake 3, for short) ${ }^{2}$, a FPS game developed by id Software and published by Activision. The choice of Quake 3 for the study was influenced by several factors. First, although Quake 3 is an older generation game (first released in December 1999), it still represents current FPS game actions in terms of perspective, weapon choices and gameplay with a sizeable player base. ${ }^{3}$ Second, Quake 3 allows command-line control of display parameters, such as the frame rate, at both load time (the game can be loaded with an initial frame rate) and at run-time via an interactive shell environment. This level of control allows for seamless switching between different control settings during the experiments. Third, in contrast to many of the current FPS games the load times for Quake 3 are relatively short. This makes it possible for a user to play numerous Quake 3 games with different frame rate settings and different maps without significant startup delays between games. This allows for study of more parameters for the same amount of user time.

\subsection{Maps}

Custom Quake 3 maps were created to allow repeated testing of the effects of frame rate for both movement and shooting actions in a short amount of time. The maps were created for the experiments using a freely available, stand-alone Quake 3 map editor.

\footnotetext{
2 http://www.idsoftware.com/games/quake/quake3-arena/.

3 A periodic sampling of GameSpy finds around 700 Quake 3 servers running on a typical weekday afternoon.
} 


\subsubsection{Movement maps}

Three maps were designed to test the primary types of movement in a FPS game: negotiating turns while walking, negotiating turns while running (running is twice the speed of walking), and jumping.

Figure 5 shows a top level view of the map designed to test turn negotiation while walking and running and Fig. 6 shows a screen capture of the same. Since the goal of the experiments is to measure the accuracy and the time taken to navigate the turns and the path under different frame rates and different movement speeds, the map was designed to:

1. Stabilize performance effects. The map is designed to reduce variability in the performance of different users. A pathway is built above a lava-like surface. Although Quake 3 has actual lava surface available, it reduces the user's health by $31 \%$ every second. This prohibits the user from falling into the lava more than three times

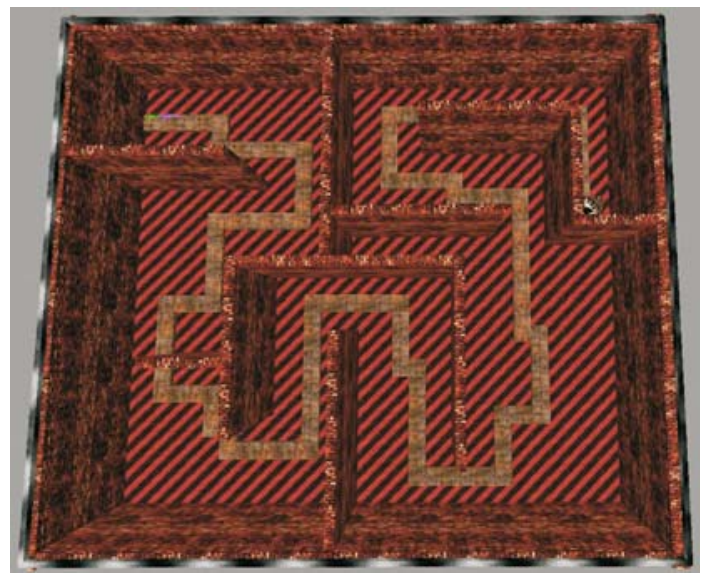

Fig. 5 Top view of the map used for negotiating turns while walking or running

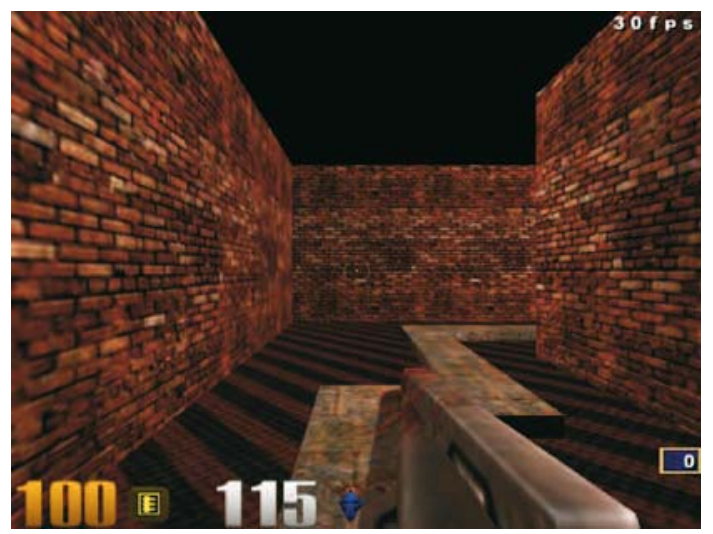

Fig. 6 Screenshot of the map used for negotiating turns while walking or running
Fig. 7 Top view of the map used for jumping

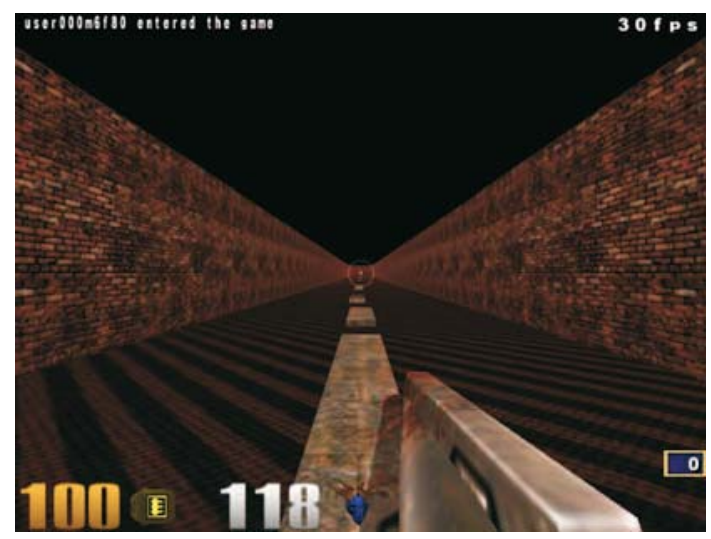

Fig. 8 Screenshot of the map used for jumping

before dying, making it difficult for some users to complete the map. Instead, a lava-like surface termed "invisible-lava", is used. The invisible lava reduces the user's health by $5 \%$ every second when stepped on.

2. Minimize wandering effects. Since the goal of the experiments is to ensure that users traverse and navigate the path as fast as possible without dying, the map was designed to ensure that users did not stray from the path or fall inadvertently. The path is lined with walls and laid out without sharp corners to discourage wandering.

3. Eliminate shooting. Although Quake 3 games are mostly played against opponents, in order to minimize the number of uncontrolled parameters, the map was designed to contain no opponents. The users were thus able to concentrate on the movement action.

Figure 7 shows a top level view of the map designed to test jumping and Fig. 8 shows a screen capture of the same. The jumping map was created by removing all turns in the map shown in Fig. 5 and replacing them with gaps of variable length so as to discourage timed jumps. Moreover, the total distance of the maps (Figs. 5 and $7^{4}$ ) was maintained at a constant.

\subsubsection{Shooting maps}

Figure 9 shows a top-level view of the shooting map and Fig. 10 shows a screen capture of the same. In keeping with the goal of the experiments-to measure shooting accuracy under different frame rates-the map was designed to:

\footnotetext{
${ }^{4}$ Figure 7 is a straightened out version of Fig. 5.
} 


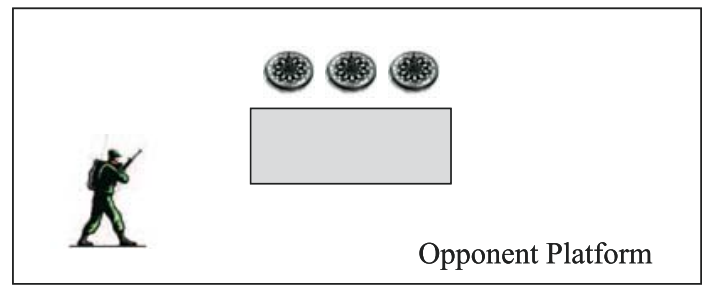

(Chasm)

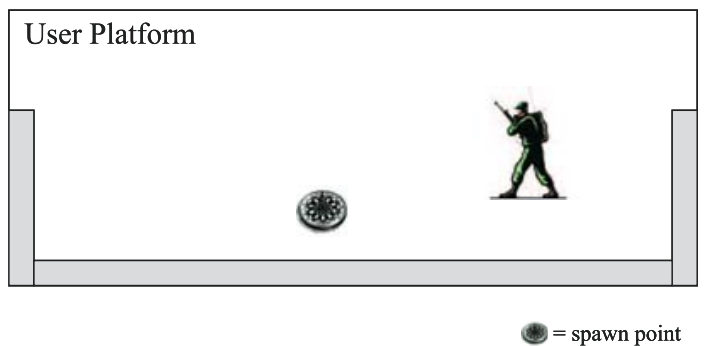

Fig. 9 Top view of the map used for shooting

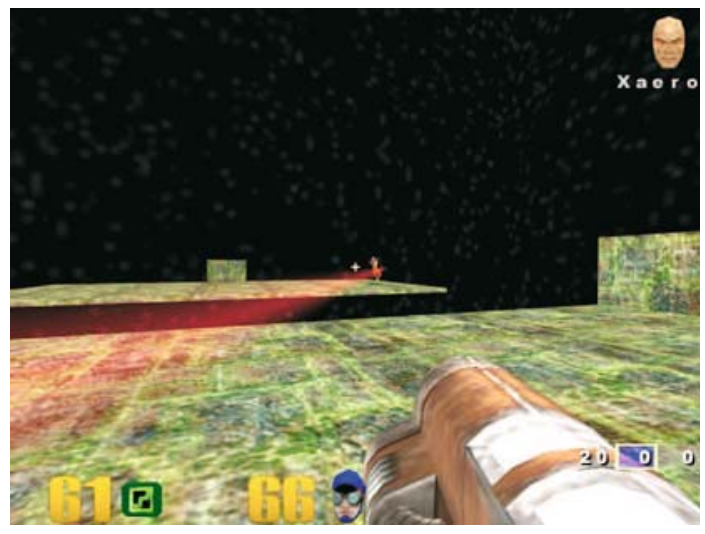

Fig. 10 Screenshot of the map used for shooting

- Minimize the uncontrollable effects of other players. In Quake 3, players are often pitted against other human players. To minimize the number of uncontrolled parameters, the user is matched against a bot-a computer controlled opponent in the map. The bot used in the map is Xaero.

- Minimize movement. Since the goal of the map is to measure the performance of shooting, the map was designed to de-emphasize the movement component of the game. The map thus comprises of two platforms divided by a chasm that cannot be jumped by the player or the bot. ${ }^{5}$

- Maximize aiming and shooting opportunities. The map was designed to ensure that the bot is always in the line of sight of the player. There are no walls or other obsta-

\footnotetext{
5 While an accidental jump or a fall into the chasm results in a death, these deaths were discarded during analysis.
}

cles that can provide cover on the bot's platform, with the exception of a single small wall that safeguards the bot's spawn point to assure that user does not pick off the bot right away. Similarly, with the exception of back and side walls to prevent accidental falls (and hence deaths), the player's platform does not have any cover.

- Minimize the effects of lighting. The map has ample light sources to ensure that dimness from poor lighting does not effect the performance of the user. Moreover, the map background is a dark sky filled with many stars, contrasting well with the more brightly colored bot, the user's target.

- Stabilize the number of shots required per kill. Higher scores (recorded by the number of kills) allow more finegrained resolution of user performance. To achieve this, the bot level in the map is set to the lowest difficulty level (level 1), and the Railgun is the only weapon available to both the player and bot. This combination allows a one-hit kill for a level 1 bot. No other weapons can be picked up by the user during the course of the game. In addition, the Railgun has a 2-s firing delay (i.e. it cannot be fired continuously), ensuring the user must actually aim and then shoot.

\subsection{Test harness}

Two separate test harnesses were used to conduct the movement and shooting experiments, respectively.

\subsubsection{Movement test harness}

The movement test harness comprised of: (1) an initial demographic survey; (2) a configuration file used to start Quake 3 with different maps and different frame rates; and (3) a data recording program that gathered the actions of the user for further analysis.

Survey. All users were asked to fill out a demographic survey prior to beginning the actual study. Figure 11 shows a snapshot of the interface to the survey. For the movement tests, no user feedback was captured between maps.

Configuration. The Quake 3 settings were pre-selected to include the three movement maps each run at three different frame rates: 3, 7, and $15 \mathrm{fps}$. Although these frame rates are lower than those that often occur on high-end PCs, even fast PCs can have episodes where computations of many players or bots can result in low frame rates. Moreover, more resource-constrained game platforms, such as hand-held devices including mobile phones, typically have lower frame rates. The frame resolution for this study was set to a resolution of $640 \times 480$, a low-end resolution for many PC games but a common resolution for console and 
Please fill in or circle the appropriate responses.

(1) Gender:

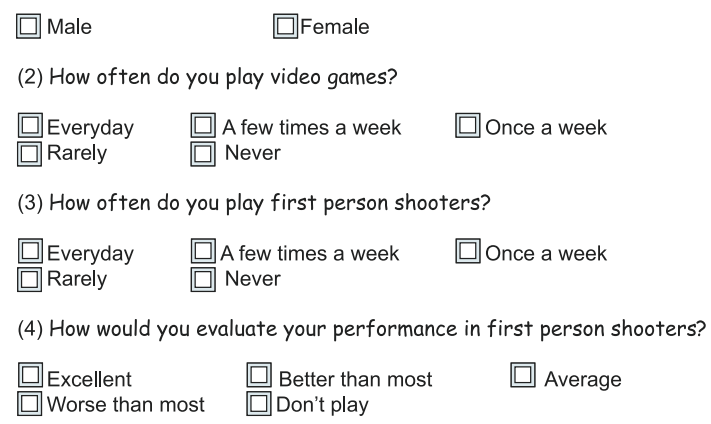

Fig. 11 User demographic survey

hand-held games. Three initial maps (one for each movement map created) at the highest resolution and frame rate were used at the start of the study to prime the user. The remainder of the maps with different frame rate settings were presented to each user in random order to mitigate any recency effects from the last played frame rate as well as any familiarity effects from repeatedly playing the same map.

Data recording. All user data was collected and recorded using FRAPS, ${ }^{6}$ a Windows application compatible with OpenGL and Direct3D technology that takes screenshots, records in-game video, and benchmarks computer games performance. FRAPS was used to record a video of the participant's performance together with a benchmark log for each map. The log recorded: the time it took the user to complete the map; the minimum, maximum, and average frame-rate of the session; and the resolution at which the participant played. FRAPS was manually started prior to the loading of each map (for each frame rate), and terminated at the end of the map by a test administrator, hidden from view by the user. By checking the video, the health remaining for each user for each map could be determined at the end of the study. Using Quake 3's built-in frame rate display, it was verified that the frame rate was not affected by the concurrent use of FRAPS.

\subsubsection{Shooting test harness}

The test harness for the shooting experiment comprised of three primary components: (1) a configuration file used to start Quake 3 with different combinations of frame rate; (2) a client program to manage the flow of game sessions and capture qualitative user comments at the end of each game; and (3) a server program to capture the statistics (deaths and kills), for each game.

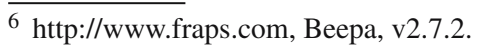

Configuration. Five different frame rates of 3, 7, 15, 30 and $60 \mathrm{fps}$ were selected. These frame rates correspond to the range of frame rates previously studied for streaming video and other interactive media applications (see Sect. 7), and also to the frame rates that appear on many game devices during normal game play. In addition, to test whether the results would hold under different resolutions, the frame resolutions were also varied for each frame rate. The frame resolutions $640 \times 480,512 \times 384$, and $320 \times 240$ were selected as representatives of resolutions used for many PC and console games down to the upper-end resolutions available in hand-held devices. The configuration file was thus pre-set to start Quake 3 with 16 different combinations of 5 frame rates and 3 frame resolutions, as well as one configuration with the highest frame rate ( 80 frames per second) and the highest resolution $(1024 \times 768)$. The highest frame rate and resolution setting was used to prime the users prior to starting the main Quake 3 runs. While the highest frame rate and resolution was the first game played by each user, all subsequent configuration combinations were presented to the user in random order to mitigate any recency effects due to the order of the display settings.

Client program. A client program was used to control the flow of the game session and to gather and record user demographics as well as user comments on the quality of the game play. User demographics were collected prior to the start of the actual experiment runs, and included gender, age group, number of hours per week of computer game play, self-rating as a gamer, and self-rating on skill level in first person shooter games. Figure 12 shows a screenshot of the interface used to gather the user demographics. The client then invoked each command of the configuration file, allowed it to run for $30 \mathrm{~s}$, and then killed the process - resulting in the user playing a specific configuration of Quake 3 for $30 \mathrm{~s}$. At the end of each $30 \mathrm{~s}$ game, users were prompted to rate the session's playability, picture quality and the effort expended in aiming and shooting the bot. In addition, users could provide free-form comments if desired. Figure 13 shows a screenshot of the

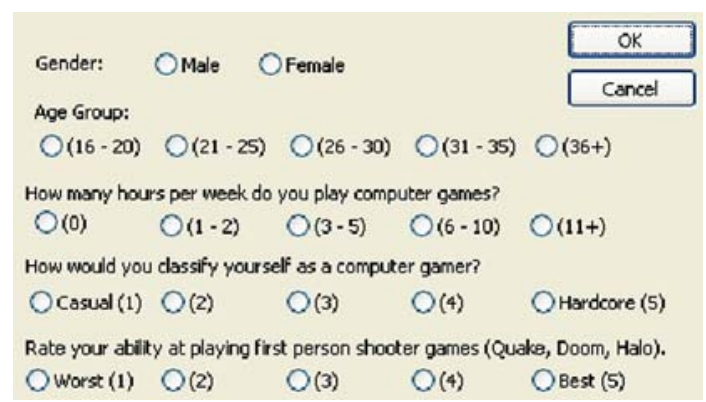

Fig. 12 Screenshot of the user demographics interface 


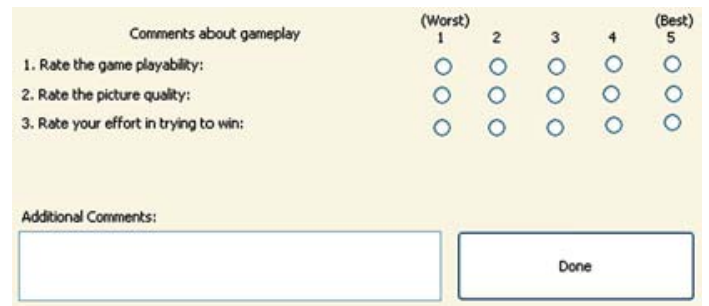

Fig. 13 Screenshot of the user comments interface

actual client interface used to record the user comments at the end of each $30 \mathrm{~s}$ game.

Statistics collector. User performance in terms of the kills and deaths was obtained from the Quake 3 server logs, while the user demographics and comment data was captured in a log produced by the Client. Users were tracked by a unique user number, but user identities were otherwise anonymized.

\subsection{Experiment environment}

The experiments were conducted in a sectioned room that enabled one person to run through the experiments without being observed by other waiting participants. Each complete run of the experiment (one user) took approximately $10 \mathrm{~min}$. and participants for the study were accepted on a first-come, first-served basis. All experiments were conducted on a Pentium 4, 2.8 GHz client with 512 MB RAM, an nVidia Geforce 6800GT 256 VRAM graphics card, and a 19" flat screen LCD monitor. A local, dedicated Pentium 4, 1.6 GHz server with 512 MB RAM ran the Quake 3 server. Both server and client ran Windows XP with service pack 2, while the Quake 3 version was point release 1.32 .

\subsection{User solicitation and demographics}

User participants for the experiments were widely solicited using a range of enticements that included: (1) a raffle for $\$ 50$ gift certificates, (2) extra credit for courses, and (3) refreshments for participants.

For the movement experiment, a total of 36 users participated, mostly computer science students or faculty. Most (77\%) were males and most (77\%) were 20-29 years old. The frequency of game play varied widely, with about a third never or rarely playing computer games and an equal number playing every day. Only one person actually played FPS games everyday, while about a third played FPS games several times a week.

For the shooting experiment, a total of 64 users took part, but data from 4 of the users was removed because they ended the client prematurely. All subsequent analysis for shooting is on the remaining 60 users that completed all frame rates and frame resolutions sets in the configuration. Most users were undergraduate computer science students in their late teens and early twenties. A sizeable number of participants (almost $25 \%$ ) were over the age of 25 , most of these being graduate computer science students. Over $65 \%$ of the users played over $1 \mathrm{~h}$ of computer games per week, with $25 \%$ playing six or more hours per week. Nearly half of the users classified themselves as casual gamers, but most classified their skills at FPS games as moderate. About $20 \%$ of the users were female. Of these, only one claimed to be more than a casual gamer, while about $65 \%$ of the males classified themselves higher than a casual gamer.

\section{Analysis}

The analysis first focuses on movement in FPS games, hypothesized in Sect. 4 to be relatively less affected by frame rate than is shooting. Section 6.1 examines walking, running and jumping. Then, the analysis focuses on shooting in Sect. 6.2, including a brief exploration of the results under different video settings and a study of user perception. The analysis ends in Sect. 6.3 with a summary comparing the relative impact of frame rate on shooting and the three movement components.

\subsection{Movement}

User performance for movement is analyzed first. One measure of performance for the movement experiments is how long it takes to complete the map by walking along the path to the end. Figure 14a depicts the effects of time to complete the map for walking. The independent variable of frame rate ranges from 3 to 15 fps for the movement experiments. Each data point represents the time averaged over all players, shown with a $95 \%$ confidence interval. Visually, the effect of frame rate on user performance shows a decrease in the amount of health loss with an increase in frame rate, with a particularly sharp drop in time from 3 to $7 \mathrm{fps}$. Statistically, the confidence intervals do not overlap for 3 and $7 \mathrm{fps}$, but they do for 7 and $15 \mathrm{fps}$. An ANOVA test shows a statistically significant difference in the three different frame rates, $F(2,95)=14.10, p<0.001$.

Another measure of performance for the movement experiments is how much health is lost due to falling off the path into the lava. The health remaining was recorded for each player at the end of each walking map. In Quake 3, players start with 120 health points and lose 1 health point per second until a health of 95 is reached. During our pilot tests, it was determined that the walking map could be completed in $10 \mathrm{~s}$ at the fastest, making the practical maximum health possible to be 110 points. Using 110 points as a max- 
Fig. 14 Effects of frame rate on time and health lost for walking. a Time versus frame rate, b health lost versus frame rate (a) Time versus Frame Rate

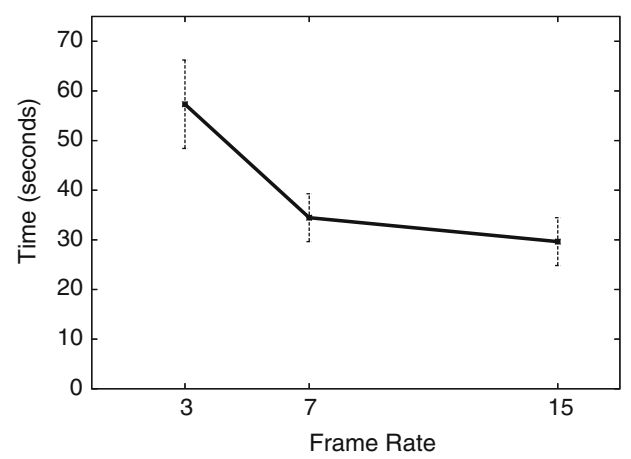

(b) Health Lost versus Frame Rate

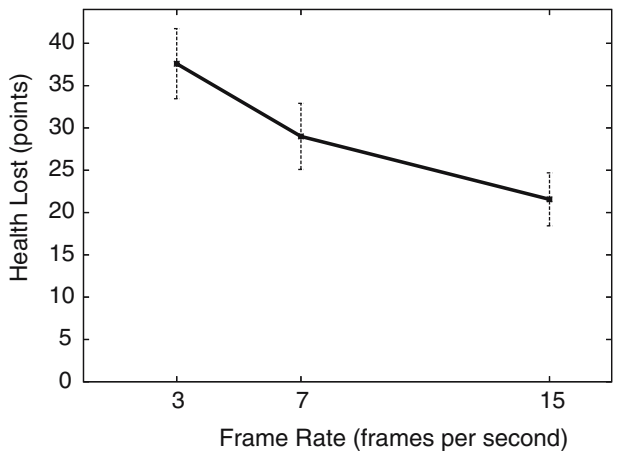

imum, Fig. 14b depicts the effects of frame rate on health lost for walking, showing average values and 95\% confidence intervals. Visually, similar to the relationship between time and frame rate, the effect of frame rate on user performance shows a decrease in the amount of health loss with an increase in frame rate. Statistically, the confidence intervals overlap for pairs of consecutive data points ( 3 and $7 \mathrm{fps}$ and 7 and $15 \mathrm{fps}$ ), but do not overlap for 3 and 15 fps. An ANOVA test shows a statistically significant difference in the three different frame rates, $F(2,95)=3.69$, $p=0.029$.

Overall, comparing the trendlines in Fig. 14a to that in Fig. 14b, frame rate degrades both movement and health lost in a visually similar way. The "knee" in the curve is higher (in terms of frame rate) for health lost than it is for time, suggesting the threshold below which frame rates increase the time for a movement related task to be somewhat lower than the threshold below which frame rates cause less accurate movement.

In order to provide measure of performance that combines health lost and time, the scores for time and health lost were combined as follows:

Score $=$ Time $\times$ Health_Lost

where lower scores are better.

Figure 15 depicts the effects of frame rate on performance (using Eq. 1) for walking, showing average values and $95 \%$ confidence intervals. Visually, the trendline is similar to Fig. 14a and b in that there is a sharp improvement in performance above $3 \mathrm{fps}$ and a more gradual improvement from 7 to $15 \mathrm{fps}$. Statistically, the confidence intervals do not overlap for any two pairs of data points. An ANOVA test shows a statistically significant difference in the three different frame rates, $F(2,95)=23.71, p<0.001$.

For brevity, analysis of the impact of frame rate on performance during running and jumping is done by examining the

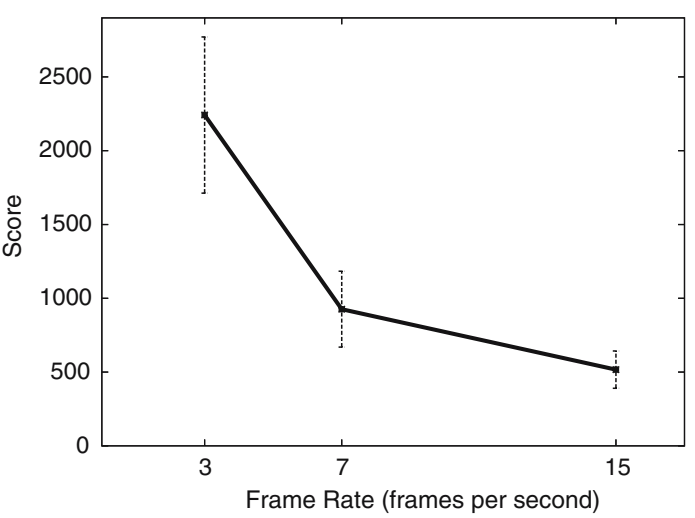

Fig. 15 Effects of frame rate on score for walking

score that combines health lost and time, with the maximum health possible adjusted as appropriate for the map.

Figure $16 \mathrm{a}$ and $\mathrm{b}$ depict the effects of frame rate on performance for running and jumping, respectively, showing average values and $95 \%$ confidence intervals. Visually, the trendlines are similar to that of walking with a sharp improvement in performance from 3 to $7 \mathrm{fps}$ and a more gradual improvement from 7 to $15 \mathrm{fps}$. Statistically, the confidence intervals for 3 and $7 \mathrm{fps}$ do not overlap, but the confidence intervals for 7 and $15 \mathrm{fps}$ do overlap. An ANOVA test shows a statistically significant difference in the three different frame rates for both running $[F(2,95)=22.40, p<0.001]$ and jumping $[F(2,95)=14.35, p<0.001]$.

\subsection{Shooting}

Performance in the shooting experiments is determined by the number of times the user killed the bot. The number of times the player was shot by the bot was also analyzed, but those results were relatively independent of the player actions and the frame rate. Thus, for all subsequent performance measurements in the shooting experiments, player 
Fig. 16 Effects of frame rate on running and jumping. a Score versus frame rate (running), b score versus frame rate (jumping) (a) Score versus Frame Rate (Running)

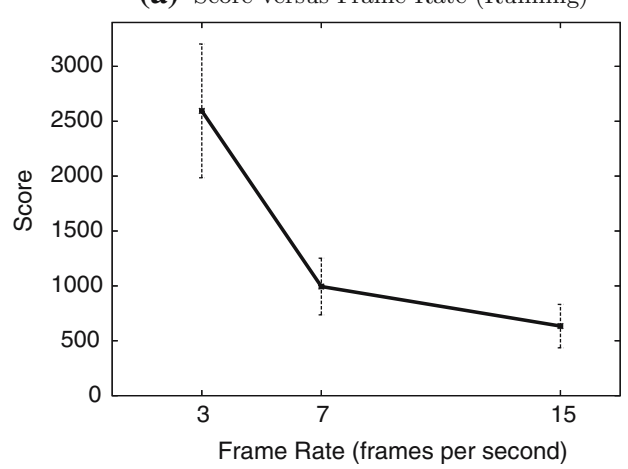

(b) Score versus Frame Rate (Jumping)

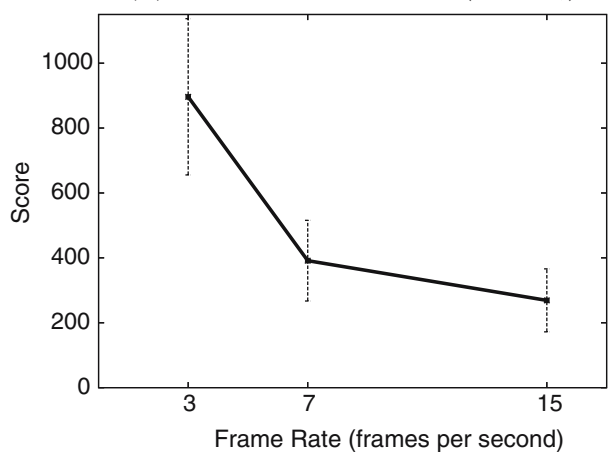

score is measured by the number of times the bot is shot in the round.

Figure 17 depicts the effects of frame rate on user performance for shooting. The independent variable of frame rate ranges from 3 to 60 for the shooting experiments. Each data point represents the average score for all players, shown with a $95 \%$ confidence interval. Visually, the effect of frame rate on user performance shows a clear logarithmic decrease in user performance with a decrease in frame rate. Statistically, the confidence intervals do not overlap for any of the data points except for 30 and $60 \mathrm{fps}$, and an ANOVA test shows a statistically significant difference in the five different frame rates, $F(4,295)=64.96, p<0.001$.

Figures $14 \mathrm{a}$ through 17 all depict performance at a frame resolution of $640 \times 480$. In order to see if the results hold for different resolutions, the analysis for the effects of frame rate on performance for shooting includes additional resolutions of $512 \times 384$ and $320 \times 240$.

Figure 18 depicts the effects of frame rate on shooting comparing three different resolutions using averages and $95 \%$ confidence intervals. Visually, the trendlines for each resolution overlap, and statistically, the confidence intervals for the averages at each frame rate also overlap. This suggests two points: (1) the effects of frame rate on performance reported

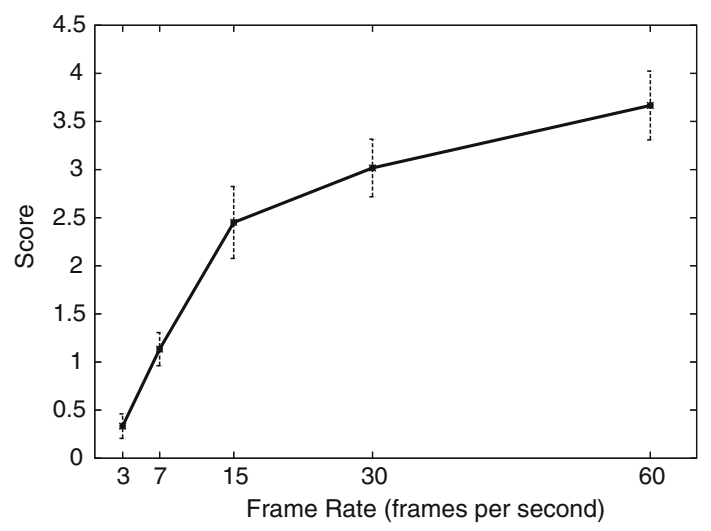

Fig. 17 Effects of frame rate on score for shooting

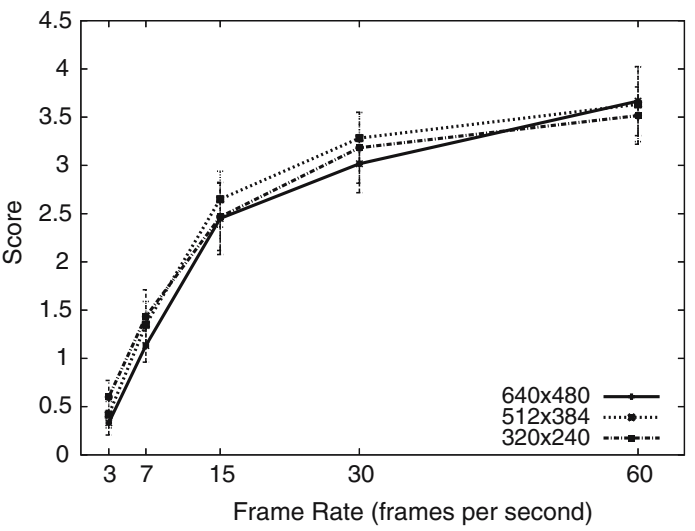

Fig. 18 Effects of frame rate on score for shooting $(640 \times 480,512 \times$ 384 and $320 \times 240$ )

in this section hold for a range of resolutions; and (2) the effect of resolution on performance is not significant compared to the effects of frame rate on performance. This last fact encourages further analysis on the effects of resolution on player performance in FPS games, but is beyond the scope of this paper and is instead our ongoing work.

While the focus of this study is on the impact of frame rate on player performance, arguably equally critical to enjoyable game play is the perceived quality of the game. The test harness for the shooting experiments gathered user opinions on the display quality and the game playability for each map played. Specifically, users rated the picture quality and the game playability on a five point scale (1 was the worst, 5 was the best).

Figure 19 depicts the effects of frame rate on players' perception of quality and playability, with the mean scores for all players shown with $95 \%$ confidence intervals. Visually, the effect of frame rate on perceived quality is clear, but less pronounced, than the effect of frame rate on user performance with a somewhat less-sharp drop in quality for lower frame rates. Statistically, the confidence intervals for the 3 and 7 fps and the 30 and 60 fps overlap, but an ANOVA test shows a significant difference between the five different levels of frame rate, $F(4,298)=138.29, p<0.001$. However, the 


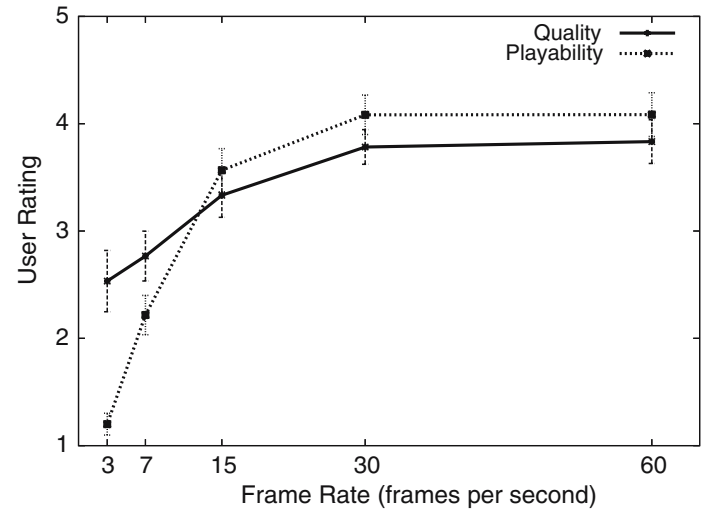

Fig. 19 Effects of frame rate on user perception

effects of frame rate on perceived playability closely follow the effects of frame rate on performance, with a sharp dropoff in playability for the lower frames and increased, but diminishing, increase in playability for higher frame rates. There is overlap in the confidence intervals at 30 and $60 \mathrm{fps}$, but an ANOVA test shows a significant difference between the five different levels of frame rate, $F(4,299)=18.97$, $p<0.001$.

\subsection{Summary}

Since the focus of this work is on the effects of frame rate on user performance in a first person shooter games, as a summary the results for shooting, walking, running and jumping area all compared. In order to compare the difference performance criteria, the scores in Figs. 15, 16a, b and 17 are normalized. The worst average performance score observed (at $3 \mathrm{fps}$ ) is set to 0 and the best average performance score of 1 is predicted to occur at $60 \mathrm{fps}$. The in-between measured data points at 7 and $15 \mathrm{fps}$ for the movement experiments and at 7, 15, and 30 fps for the shooting experiments are smoothed with a Bezier curve. Figure 20b depicts the results.
Figure 20a shows the normalized relationship between the three movement tasks: walking, running and jumping. The effects of frame rate on performance are quite similar for all movement tasks, suggesting the precision and deadline for moving in a FPS game is fundamentally related to the first-person perspective and not the speed or type of movement. Averaging the walking, running and jumping curves into one curve called "movement", Fig. 20b shows the normalized comparison of movement and shooting. Frame rate has a larger effect on shooting performance than it does on movement performance, as evidenced by the sharper drop-off in performance as frame rates decrease. Movement performance is more resilient to lower frame rates, and the knee in the movement curve is around $17 \mathrm{fps}$ while the knee in the shooting curve is around $25 \mathrm{fps}$.

\section{Related work}

Previous research that is related to the work in this paper is divided broadly into two categories: (1) research with active users that explore the impact of various visual degradations on user performance; and (2) research with passive users that examine the perceived quality of video as it is being watched under different display settings. The studies with active users are more relevant to our work since our user studies involve players actively playing a FPS game.

\subsection{Active users}

Swartz and Wallace [18] examined the effects of frame rate and resolution on skilled users tasked with identifying, tracking and designating targets using unmanned aerial vehicles. The users' tasks were accomplished by watching a short video clip in the first study and flying a vehicle in a simulator in the second study. The independent variables were frame rates of 2, 4 and $7.5 \mathrm{fps}$ and resolutions of 2, 8 and 12 lines on the television set. While the effects of frame rate
Fig. 20 Predicted effects of frame rate on performance. a Walking, running and jumping versus frame rate, $\mathbf{b}$ movement and shooting versus frame rate (a) Walking, Running and Jumping versus Frame Rate

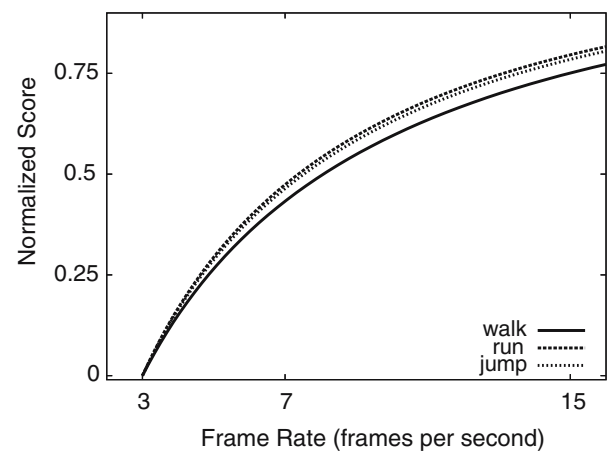

(b) Movement and Shooting versus Frame Rate

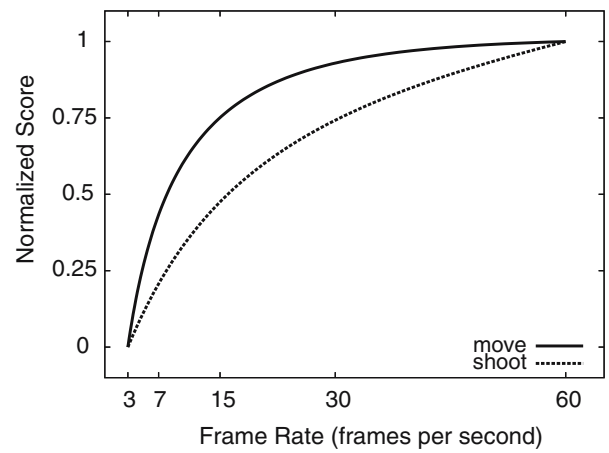


were statistically significant, there was minimal difference between performance of 4 or $7.5 \mathrm{fps}$ and the authors suggest $4 \mathrm{fps}$ is enough for acceptable performance. Resolution had only marginal effects overall on task performance although the effects on image quality ratings were significant.

Smets and Overbeeke [17] explored the trade-off between frame rate, resolution and interactivity for users solving simple spatial puzzles with their hands. Digital cameras showing the users hands and puzzle were fed through a computer that modified the resolution and then fed the image to a headmounted display worn by the users. The amount of interactivity was controlled by the location of the camera, being either head-mounted or fixed to the side of the puzzles. The independent variables were resolutions of $768 \times 576,36 \times 30$ and $18 \times 15$, with frame rates of 25 and 5 fps controlled by the user of a stroboscopic light. Frame rate was not a statistically significant factor in performance while the main effects of resolution were statistically significant. Although their analysis included generally appropriate statistical tests (ANOVA), they had only four users making the generality of their results suspect.

Massimo and Sheridan [12] studied the performance of tele-operation with varying force of feedback, task difficulty and frame rates. Six users, all graduate students from MIT, remotely operated a mechanical arm to complete a puzzle requiring the placement of pegs in holes. In one study, users observed the puzzle via a remotely operated video link with video frame rates of 3,5 and $30 \mathrm{fps}$, all with a resolution of $512 \times 256$ pixels. The effects of frame rate on user performance (the time to complete the puzzle) were found to be statistically significant, with a large change in performance from 3 to 5 fps and a smaller change in performance from 5 to $30 \mathrm{fps}$. Interestingly, the presence of force feedback, not commonly available in computer games, was able to make up for any deficiencies in performance at $3 \mathrm{fps}$. Although they claimed their results confirm early research, the small number of users in their study (6) calls into question the generality of their results.

These studies are significant in that they suggest users can tolerate low frame rates and still achieve acceptable performance. Our study differs primarily in that our group of users is substantially larger, and our users interact with a virtual environment over a wider range of frame rates that are available in today's interactive gaming environments.

\subsection{Passive users}

There have been a number of studies measuring the reaction of users to passively watching videos with various frame rates.

McCarthy et al. [13] examined the percentage of time sports videos at varying frame rates and resolutions was acceptable to users in the context of streaming to small screen devices, such as mobile phones. Users watched sport videos in which the frame rate and/or frame resolution were gradually degraded until the users indicated the quality was not acceptable. Contrary to earlier findings, the authors found users preferred higher resolutions to higher frame rates, and found frame rates as low as $6 \mathrm{fps}$ were acceptable $80 \%$ of the time.

Ghineas and Thomas [9] looked at the effects of frame rate on the ability of users to understand the content of video clips. Users observed videos selected from various categories at 5 , 15 and 25 fps and answered questions pertaining to their content. Analysis found that even the lowest frame rates (5 fps) did not result in any significant loss in information content.

Apteker et al. [1] studied the effects of frame rates on the watchability of videos. Users watched and rated eight videos with varying spatial and temporal characteristics, at 5,10 and $15 \mathrm{fps}$ on a display of $160 \times 120$ pixels. The effects of frame rate on the watchability of the videos was statistically significant but the effects of the lowest frame rate, $5 \mathrm{fps}$, did not result in a marked decrease in watchability for all videos.

Tripathi and Claypool [19] studied the impact of frame rate and resolution on videos with different content, specifically high-motion videos and low-motion videos. Users watched and rated the perceived quality of several short video clips degraded by either a reduced frame rate or a reduced frame resolution. The authors found that the effects of decreasing the frame rate and resolution depended upon the motion content, with high-motion videos appearing more degraded with a decrease in frame rate and low-motion videos appearing more degraded more with a decrease in resolution.

Johnson and Caird [11] examined the degree to which users can recognize sign language over degraded frame rates. Forty-eight users were trained in sign language and then watched videos of sign language gestures at 1, 5, 15 and 30 fps. Analysis of the data included ANOVA tests and showed participants were able to learn to recognize signs even at the lowest frame rates.

Bryson [3] examined the effects of delay and frame rate on the ability to track an object on the screen. Two types of tracking were studied: pursuit tracking where an object is followed on the screen with a pointer and pursuit tracking where a pointer is moved from one location to another. Users used a custom application that measured accuracy and time for the tracking tasks using a mouse. Overall, the author suggested that low frame rates quantitatively impact performance similarly to delay. However, only two users participated in the study so the conclusions may not be statistically valid.

These studies are significant in that they show that at least for some videos, users can tolerate video displayed at low frame rates and that users generally prefer higher resolutions. Our work differs in that computer games, in general, and FPS games, specifically, are highly interactive. 


\section{Conclusion}

The growth in the diversity of technology to support computer games brings an increasing need for a better understanding of the impact of frame rate on game players. This paper presents analysis from two large user studies designed to measure the effects of frame rate on the fundamental interactions for users playing a FPS game. One-hundred users participated in two sets of experiments, providing user performance and perception data over a range of frame rates commonly studied for video streaming and inclusive of frame rates found in many computer game platforms.

Analysis of the performance results shows that for the ranges tested, frame rate has a larger impact on performance than typically found for streaming video or even other interactive multimedia applications. Moreover, a suitable frame rate, in particular, is critical for adequate game performance. Frame rates as low as $3 \mathrm{fps}$ and even $7 \mathrm{fps}$ are almost unplayable as users cannot adequately target opponents. In fact, there are performance benefits for user play up through $60 \mathrm{fps}$, where a frame rate of $60 \mathrm{fps}$ provides a seven fold increase in shooting performance over a frame rate of $3 \mathrm{fps}$. While frame rate is also important for player movement, the degradation in performance for movement related tasks from decreased frame rates does not drop as early as for shooting related tasks. These results hold across multiple screen resolutions.

Analysis of the users' perceptions shows that the effect of frame rate on perceived quality has an effect similar to, if less pronounced, the effect of frame rate on performance, with less of a difference in perceptual quality for the higher frame rates. However, the effects of frame rate on perceived playabilty closely mirror the measured effects of frame rate on performance.

The overall results are dramatically different than those obtained for previous research that assessed the effects of frame rate for streaming video. Those studies concluded that even moderate frame rates were not critical for acceptable performance, with frame rates as low as 7 and perhaps even 3 fps being acceptable. For FPS games, frame rate has a major impact on user performance, suggesting frame rates should be preserved even if it means sacrificing display resolution. This contrast suggests there may be challenges in designing devices that can effectively support both computer games and streaming multimedia as the quality of service (QoS) for computer games appears to be significantly different than for other forms of multimedia.

\section{Future work}

The results presented in this paper were obtained by user studies performed for particular maps played under varying frame rates. Our ongoing work is to explore the effects of frame resolution on user performance in FPS games, leveraging the test harnesses and methodology developed for this study. In addition, environmental details for FPS games such as different maps, light versus dark backgrounds and different types of guns pose interesting future work. In particular, guns with different precision and deadline requirements should show somewhat different degradations under lowered frame rates. Moreover, while it is hoped that the results obtained in this study for FPS games generalize to other computer game genres, future work could include a study similar to the one presented in this paper for Real-Time Strategy games, 3D action games, or sports games.

Acknowledgments We would like to acknowledge the help of Tim Connor, Feissal Daama, Adam Fiske and Ryan Kennedy for their help in running the experiments and for the many users that participated in the user studies - their help provided the data that made the analysis in this paper possible.

\section{References}

1. Apteker, R.T., Fisher, J.A., Kisimov, V.S., Neishlos, H.: Video Acceptability and Frame Rate. IEEE Multimedia, pp. 32-40 (1995)

2. Beigbeder, T., Coughlan, R., Lusher, C., Plunkett, J., Agu, E., Claypool, M.: The effects of loss and latency on user performance in Unreal Tournament 2003. In: Proceedings of ACM Network and System Support for Games Workshop (NetGames), Portland, OG, USA (2004)

3. Bryson, S.: Effects of lag and frame rate on various tracking tasks. In: Proceedings of SPIE Stereoscopic Displays and Applications, vol. 1915, pp. 155-166 (1993)

4. Claypool, K., Claypool, M.: The effects of resolution on users playing First Person Shooter games. In: Proceedings ACM/SPIE Multimedia Computing and Networking (MMCN) Conference, San Jose, CA, USA (2007)

5. Claypool, M.: The effect of latency on user performance in RealTime Strategy games. Elsevier Comput. Netw. Spec. Issue. Netw. Issues Entertain. Comput. 49(1), 52-70 (2005)

6. Claypool, M., Claypool, K.: Latency and Player Actions in Online Games. Commun. ACM 49(11), 40-45 (2006)

7. Dick, M., Wellnitz, O., Wolf, L.: Analysis of factors affecting players' performance and perception in multiplayer games. In: Proceedings of the 4th ACM Network and System Support for Games (NetGames), Hawthorne, NY, USA (2005)

8. Fritsch, T., Ritter, H., Schiller, J.H.: The effect of latency and network limitations on MMORPGs: a field study of Everquest 2. In: Proceedings of the 4th ACM Network and System Support for Games (NetGames), Hawthorne, NY, USA (2005)

9. Ghinea, G., Thomas, J.P.: QoS impact on user perception and understanding of multimedia video clips. In: Proceedings of ACM Multimedia Conference, Bristol, UK (1998)

10. Henderson, T., Bhatti, S.: Networked games-a QoS-sensitive application for QoS-insensitive users? In: Proceedings of the ACM SIGCOMM Workshop on Revisiting IP QoS, pp. 141-147, Karlsruhe, Germany (2003)

11. Johnson, B.F., Caird, J.K.: The effect of frame rate and video information redundancy on the perceptual learning of American sign language gestures. In: Conference on Human Factors in Computing Systems, pp. 121-122, Vancouver, BC, Canada (1996) 
12. Massimino, M.J., Sheridan, T.B.: Teleoperator performance with varying force and visual feedback. Hum. Factors, 36, 145157 (1994)

13. McCarthy, J., Sasse, M.A., Miras, D.: Sharp or smooth? Comparing the effects of quantization versus frame rate for streamed video. In: Proceedings of ACM CHI Conference on Human Factors in Computing Systems, Vienna, Austria (2004)

14. Nichols, J., Claypool, M.: The effects of latency on online Madden NFL football. In: Proceedings of the 14th ACM International Workshop on Network and Operating Systems Support for Digital Audio and Video (NOSSDAV), Kinsale, County Cork, Ireland (2004)

15. Pantel, L., Wolf, L.C.: On the impact of delay on real-time multiplayer games. In: Proceedings of the Workshop on Network and Operating Systems Support for Digital Audio and Video (NOSSDAV), Miami, FL, USA (2002)

16. Reddy, M.: The effects of low frame rate on a measure for user performance in virtual environments. Technical report ECS-CSG36-97, Department of Computer Science, University of Edinburgh (1997)

17. Smets, G., Overbeeke, K.: Trade-off between resolution and interactivity in spatial task performance. IEEE Comput. Graph. Appl. pp. 46-51 (1995)
18. Swartz, M., Wallace, D.: Effects of frame rate and resolution reduction on human performance. In: Proceedings of IS\&T's 46th Annual Conference, Munich, Germany (1993)

19. Tripathi, A., Claypool, M.: Improving multimedia streaming with content-aware video scaling. In: Workshop on Intelligent Multimedia Computing and Networking (IMMCN), Durham, NC, USA (2002)

20. van den Branden Lambrecht, C.J., Verscheure, O.: Quality measure using a spatio-temporal model of the human visual system. In: Proceedings of SPIE, San Jose, CA, USA (1996)

21. Webster, A.A., Jones, C.T., Pinson, M.H., Voran, S.D., Wolf, S.: An objective video quality assessment system based on human perception. In: Proceedings of SPIE Human Vision, Visual Processing, and Digital Display IV, San Jose, CA, USA (1993)

22. Zebarth, J.A.: Let me be me. In: Proceedings of Globecom-IEEE Telecommunications Conference, pp. 389-393, Houston, TX, USA (1993)

23. Zink, M., Kunzel, O., Schmitt, J., Steinmetz, R.: Subjective impression of variations in layer encoded videos. In: Proceedings of the 11th International Workshop on Quality of Service (IWQoS), Monterey, CA, USA (2003) 